PROCEEDINGS OF THE

AMERICAN MATHEMATICAL SOCIETY

Volume 127, Number 1, January 1999, Pages 51-55

S 0002-9939(99)04823-6

\title{
FINITELY GENERATED GROUP RING UNITS
}

\author{
ŁUKASZ WIECHECKI
}

(Communicated by Ronald M. Solomon)

\begin{abstract}
We give a classification of nilpotent groups $G$ for which the unit group of the integral group ring is finitely generated.
\end{abstract}

For a group $G$ let $\mathcal{U} \mathbb{Z} G$ denote the group of invertible elements in the integral group ring $\mathbb{Z} G$. This is a subject of intensive study; see Sehgal [5].

The natural question arises as to when $\mathcal{U} \mathbb{Z} G$ is finitely generated. It is easy to see that if this is the case, then $G$ must be finitely generated. Yet the question has remained unanswered even for the (simplest nonabelian) case of nilpotent groups. In the literature one can find a few partial results. The first is

1. Theorem (Sehgal [4], p. 194). Suppose that $G$ is an extension of a torsion group $T$ by a torsion free nilpotent group. If $\mathbb{Z} G$ has no nilpotent elements, then $\mathcal{U} \mathbb{Z} G=$ $(\mathcal{U} \mathbb{Z} T) \cdot G$.

It is well known that $\mathcal{U} \mathbb{Z} T$ is finitely generated for any finite group $T$ and hence Theorem 1 implies that $\mathcal{U} \mathbb{Z} G$ is finitely generated if $\mathbb{Z} G$ contains no nilpotent elements. On the other hand we have a result obtained recently by Marciniak and Sehgal [2] who proved that $\mathcal{U} \mathbb{Z} G$ is not finitely generated for $G=\mathbb{Z} \times D_{8}$, where $D_{8}$ is the dihedral group of order 8. Another result of this sort is due to Mirowicz [3]. He showed that $\mathcal{U} \mathbb{Z}\left[D_{\infty}\right]$ is not finitely generated for the infinite dihedral group $D_{\infty}$

In the present paper we determine when $\mathcal{U} \mathbb{Z} G$ is finitely generated for nilpotent groups $G$. In what follows we denote by $T(G)$ the set of torsion elements in $G$ and by $\mathcal{Z}(G)$ the center of $G$. It is well known for finitely generated nilpotent groups $G$ that $T(G)$ is a finite characteristic subgroup which is the product of its Sylow subgroups. For the details concerning nilpotent groups we refer the reader to [1]. Our main result reads:

2. Theorem. Let $G$ be a nilpotent group. Then $\mathcal{U} \mathbb{Z} G$ is finitely generated if and only if either $G$ is finite or $G$ is finitely generated and there are no nilpotent elements in $\mathbb{Z} G$.

By using Sehgal's characterization of group rings which have no nilpotent elements (see Theorem 10) one can formulate this result in purely group theoretic terms as follows.

Received by the editors May 12, 1997.

1991 Mathematics Subject Classification. Primary 16S34, 20 C07.

(C)1999 American Mathematical Society 
3. Corollary. Let $G$ be a nilpotent group. Then $\mathcal{U} \mathbb{Z} G$ is finitely generated if and only if either $G$ is finite or $G$ is finitely generated, all finite subgroups of $G$ are normal and its torsion part $T(G)$ satisfies one of the following conditions:

(i) $T(G)$ is abelian, or

(ii) $T(G)=A \times E \times K_{8}$ where $E$ is an elementary abelian 2-group and $A$ is a finite abelian group of odd order $m$ such that the multiplicative order of $2 \bmod m$ is odd.

We start with four auxiliary results.

4. Lemma. Let $G$ be a nilpotent group with a finite normal p-subgroup $H$. Then the ideal $\Delta(G, H)=\operatorname{ker}\left(\mathbb{F}_{p} G \rightarrow \mathbb{F}_{p}[G / H]\right)$ is nilpotent.

Proof. We proceed by induction on $|H|$. If $H=\left\langle t \mid t^{p}=1\right\rangle$, then

$$
\Delta(G, H)=\left(\mathbb{F}_{p} G\right)(t-1)=(t-1)\left(\mathbb{F}_{p} G\right)
$$

and hence for any $\alpha_{i}=\beta_{i}(t-1) \in \Delta(G, H)(1 \leq i \leq p)$ we have $\alpha_{1} \cdots \alpha_{p} \in$ $\mathbb{F}_{p} G(t-1)^{p}=\mathbb{F}_{p} G\left(t^{p}-1\right)=0$. Therefore the lemma is true when $|H|=p$.

Suppose now $|H|=p^{n}, n>1$. As $G$ is nilpotent and $H \triangleleft G$, so $H \cap \mathcal{Z}(G) \neq 1$ and hence there exists a central subgroup $C \triangleleft H$ with $|C|=p$. For $\phi: \mathbb{F}_{p} G \rightarrow$ $\mathbb{F}_{p}[G / C]$ we clearly have $\phi(\Delta(G, H)) \subseteq \Delta(G / C, H / C)$. By the inductive hypothesis, $\Delta(G / C, H / C)$ and $\Delta(G, C)$ are nilpotent, hence

$$
(\Delta(G, H))^{s t} \subseteq(\Delta(G, C))^{t}=0
$$

for suitable $s, t$.

5. Lemma. Let $G$ be a finitely generated nilpotent group of Hirsch rank 1. Then $G$ is a semidirect product of its finite subgroup $T(G)$ with an infinite cyclic group.

Proof. $G / T(G)$ is a finitely generated torsion free nilpotent group of Hirsch rank 1 , so it is infinite cyclic.

6. Lemma. If $G$ is a finitely generated nilpotent group with an infinite and normal subgroup $H$, then there exists a subgroup $H_{1}<H, H_{1} \triangleleft G$, such that $H / H_{1} \simeq \mathbb{Z}$.

Proof. Consider the upper central sequence of $G$ : $\zeta_{0} G<\zeta_{1} G<\zeta_{2} G<\cdots$ and the subgroups $D_{j}=\zeta_{j} G \cap H$. Let $i$ be the (least) integer satisfying $\left|H / D_{i}\right|=\infty$ and $\left|H / D_{i+1}\right|<\infty$. Then $D_{i+1} / D_{i}$ is infinite, finitely generated and lies in the center of $G / D_{i}$. Besides, $\left|\left(H / D_{i}\right):\left(D_{i+1} / D_{i}\right)\right|<\infty$. Thus we can find a group $D_{i+\frac{1}{2}}$ such that $D_{i} \leq D_{i+\frac{1}{2}}<D_{i+1}$ and $D_{i+1} / D_{i+\frac{1}{2}} \simeq \mathbb{Z}$. From Lemma 5 it follows that $H / D_{i+\frac{1}{2}}$ is a semidirect product of $\mathbb{Z}$ with its subgroup $T$ of torsion elements which, by the way, is normal in $G / D_{i+\frac{1}{2}}$. Hence we may define

$$
H_{1}=\pi^{-1}(T)
$$

where $\pi: G \rightarrow G / D_{i+\frac{1}{2}}$ is the natural homomorphism.

7. Lemma. Let $R$ be a ring of characteristic $p$ and $J$ its nilpotent ideal. Then $1+J$ is a nilpotent p-group.

Proof. If $y \in J$, then $(1+y)^{-1}=1-y+y^{2}-\cdots \in 1+J$, hence $1+J$ is a group. Next observe that $\left[1+J^{k}, 1+J^{l}\right] \subseteq 1+J^{k+l}$. Indeed, if $a \in J^{k}$ and $b \in J^{l}$, then $[1-a, 1-b]=\left(1+a+a^{2}+\cdots\right)\left(1+b+b^{2}+\cdots\right)(1-a)(1-b)=1+($ a sum of products in which both $a$ and $b$ occur $) \in 1+J^{k+l}$. So our inclusion holds and 
consequently $1+J$ is a nilpotent group. To prove that $1+J$ is a $p$-group notice that if $a \in J$, then $(1+a)^{p^{n}}=1+a^{p^{n}}=1$ for $n$ sufficiently large.

Definition. A group ring unit $u \in \mathbb{Z} G$ is unipotent if $(u-1)^{n}=0$ for some $n \geq 1$.

Definition. A subgroup $U<\mathcal{U} \mathbb{Z} G$ is $p$-nice if $\mathcal{Z}(G)<U$ and $G$ has a finite normal $p$-subgroup $V$ such that the image of $U$ in $\mathbb{Z}[G / V]$ consists of trivial units only.

8. Theorem. Suppose $U<\mathcal{U} \mathbb{Z} H$ is p-nice and the center $\mathcal{Z}(H)$ is of finite index in $H$. If $U$ is finitely generated, then any infinite set of unipotent units in $U$ has two elements $u, v$ such that $p \mid(u-v)$.

Proof. We have two commuting diagrams of natural maps:
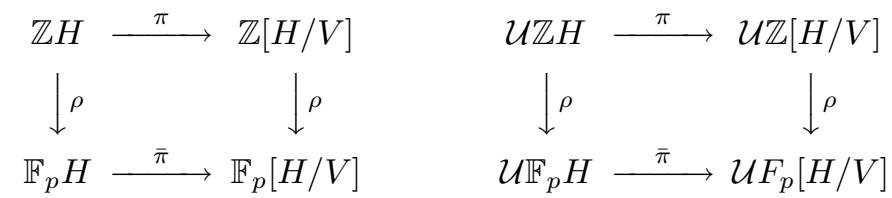

Let $\bar{U}=\rho(U)<\mathcal{U} \mathbb{F}_{p} H$ and $I=\operatorname{ker}\left(\bar{\pi}: \mathbb{F}_{p} H \rightarrow \mathbb{F}_{p}[H / V]\right)=\Delta(H, V)$. As $\bar{\pi}(\bar{U})=$ $\rho \pi(U)< \pm H / V$, so we have an exact sequence

$$
1 \rightarrow(1+I) \cap \bar{U} \rightarrow \bar{U} \rightarrow \pm H / V .
$$

Let $Z=\mathcal{Z}(H)$. We obtain an exact sequence

$$
1 \rightarrow((1+I) \cap \bar{U}) /((1+I) \cap \bar{U} \cap Z) \rightarrow \bar{U} / Z \rightarrow \pm H /(Z \cdot V)
$$

with $H /(Z \cdot V)$ finite. As $\bar{U} / Z$ is finitely generated, so is $((1+I) \cap \bar{U}) /((1+I) \cap \bar{U} \cap Z)$. But $(1+I) \cap \bar{U} \cap Z<(1+I) \cap Z<V$, hence $(1+I) \cap \bar{U} \cap Z$ is finite. Therefore $(1+I) \cap \bar{U}$ is finitely generated. By Lemmas 4 and 7 it is a nilpotent $p$-group, hence it is finite.

Let $S \subseteq U$ be an infinite set of unipotent units. As 1 is the only unipotent unit in $\pm H / V \subset \mathbb{Z}[H / V]$ so $\pi(u)=1$ for all $u \in S$. Hence $\bar{\pi} \rho(u)=\rho \pi(u)=1$ and $\rho(u) \in(1+I) \cap \bar{U}$. It follows that $\rho(u)=\rho(v)$ for some $u, v \in S$.

For an element $\alpha \in \mathbb{Z} H$ let $C(\alpha)$ denote the centralizer of $\alpha$ in $H$, i.e., $C(\alpha)=$ $\{h \in H: h \alpha=\alpha h\}$.

9. Proposition. Suppose $\mathbb{Z} G$ contains a nilpotent element $\eta \neq 0$. If there exist a group $H$ with $|H: \mathcal{Z}(H)|<\infty$ and a homomorphism $\phi: G \rightarrow H$ such that $\phi(\eta) \neq 0$, $|\phi(C(\eta))|=\infty$ and $\phi(\mathcal{U} \mathbb{Z} G)$ is p-nice in $\mathcal{U} \mathbb{Z} H$, then $\mathcal{U} \mathbb{Z} G$ is not finitely generated.

Proof. We may assume that not all coefficients of $\eta$ are divisible by $p$. Set $U=$ $\phi(\mathcal{U} \mathbb{Z} G)$. Let $h_{1}, h_{2}, \ldots \in C(\eta)$ map to different elements in $H$. Consider $X=$ $\left\{1+h_{i} \eta: i=1,2, \ldots\right\} \subseteq \mathcal{U} \mathbb{Z} G$. Let overbar denote images under $\phi$. If $u=1+\bar{h}_{i} \bar{\eta}$, $v=1+\bar{h}_{j} \bar{\eta} \in U$, then $u-v=\left(\bar{h}_{i}-\bar{h}_{j}\right) \bar{\eta}$. Now, for every $h_{i}$ we are able to choose infinitely many elements $h_{j_{1}}, h_{j_{2}}, \ldots$ such that $\operatorname{supp}\left(\bar{h}_{i} \bar{\eta}\right) \cap \operatorname{supp}\left(\bar{h}_{j_{k}} \bar{\eta}\right)=\varnothing$ and hence $p \nmid\left(\bar{h}_{i}-\bar{h}_{j_{k}}\right) \bar{\eta}$. Carrying out the standard diagonal procedure we can find an infinite subset $S$ of $X$ such that $p \nmid(u-v)$ for every $u, v \in S$. Our proposition now follows from Theorem 8 .

We now use Theorem 8 to classify finitely generated nilpotent groups $G$ for which $\mathcal{U} \mathbb{Z} G$ is not finitely generated. Recall first the following 
10. Theorem (Sehgal [4], p. 176). Let $G$ be a finitely generated nilpotent group. The group ring $\mathbb{Z} G$ has no nilpotent elements if every finite subgroup of $G$ is normal and either

(i) $T(G)$ is abelian, or

(ii) $T(G)=A \times E \times K_{8}$ where $E$ is an elementary abelian 2-group and $A$ is a finite abelian group of odd order $m$ such that the multiplicative order of $2 \bmod m$ is odd.

Now, using Theorems 8 and 10, we prove the following

11. Theorem. If $G$ is an infinite nilpotent group and $\mathbb{Z} G$ contains a nonzero nilpotent element, then $\mathcal{U} \mathbb{Z} G$ is not finitely generated.

Proof. If $\mathcal{U} \mathbb{Z} G$ is generated by $u_{1}, \ldots, u_{m}$, then $G$ is generated by $\bigcup_{i=1}^{m} \operatorname{supp}\left(u_{i}\right)$. Therefore we can assume that $G$ is finitely generated. We know that every finitely generated nilpotent group is torsion free-by-finite so we can find a normal torsion free subgroup $H_{1} \triangleleft G$ such that $\left|G: H_{1}\right|<\infty$. Lemma 6 assures the existence of a normal in $G$ subgroup $H_{2}<H_{1}$ such that $H_{1} / H_{2} \simeq \mathbb{Z}$. Then the Hirsch rank of $G / H_{2}$ is 1 .

From Theorem 10 it follows that we are to consider two cases. First let us assume that there exists a finite subgroup $E<G$ which is not normal. As $T(G)$ is the product of its Sylow subgroups we may assume that $E=\langle y\rangle$ where $o(y)=p^{n}$ for some prime $p$. We can always find an element $t \in G$ such that $t^{-1} E t \neq E$ and $o(\bar{t})=\infty$ where $\bar{t}$ is the image of $t$ in $G / H_{2}$. This is so because $\left[T(G), H_{2}\right]=1$ and for any elements $z, x \in G$ such that $o(\bar{z})=\infty$ and $x^{-1} E x \neq E$ one of the elements $x, x z, z$ fits. Let $T(G)=S_{p} \times S_{p^{\prime}}$ where $S_{p}$ is the Sylow $p$-subgroup of $T(G)$.

Now we put: $H=G /\left(H_{2} \cdot S_{p^{\prime}}\right), \phi: G \rightarrow H$ is the natural homomorphism, $\eta=(1-y) t\left(1+y+\cdots+y^{p^{n}}-1\right), V=\phi\left(S_{p}\right) \simeq S_{p}, U=\phi(\mathcal{U} \mathbb{Z} G)$ and check that all assumptions of Proposition 9 are satisfied.

We have the following diagram of natural homomorphisms:

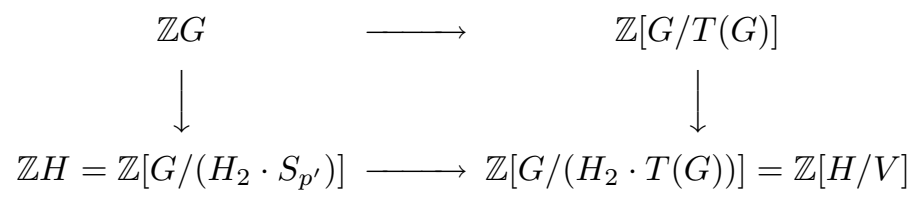

and $\mathbb{Z}[G / T(G)]$ has only trivial units because $G / T(G)$ is torsion free nilpotent. Also $H<U$, hence $U$ is $p$-nice. It is clear that $\phi(\eta) \neq 0$. As $T(G)$ is finite we can find an integer $k$ such that $t^{k}$ commutes with $T(G)$ and from the construction of $\eta$ it follows that we have an infinite subset $\left\{\bar{t}^{k l}: l=1,2, \ldots\right\} \subseteq \phi(C(\eta))$. Finally, $\mid H$ : $\mathcal{Z}(H) \mid<\infty$, because $H$ has Hirsch rank 1 . Hence all assumptions of Proposition 9 are satisfied and so $\mathcal{U} \mathbb{Z} G$ is not finitely generated.

Let us now suppose that every finite subgroup of $G$ is normal. As $\mathbb{Z} G$ contains a nonzero nilpotent element we can, by Theorem 10, assume that $T(G)=A \times E \times K_{8}$, where $E$ is an elementary abelian 2-group and $A$ is a finite abelian group of odd order $m$ such that the multiplicative order of $2 \bmod m$ is even. Recall that we have found torsion free normal subgroups $H_{1}$ and $H_{2}$ such that $G / H_{1}$ is finite and $H_{1} / H_{2} \simeq \mathbb{Z}$.

Let $|A|=m$. If $m=p_{1}^{\alpha_{1}} p_{2}^{\alpha_{2}} \cdots p_{k}^{\alpha_{k}}$, then there is at least one $1 \leq i \leq k$ such that the multiplicative order of $2 \bmod p_{i}^{\alpha_{i}}$ is even. Indeed, otherwise $2^{l_{i}} \equiv 1\left(\bmod p_{i}^{\alpha_{i}}\right)$ for some odd integers $l_{i}$. But then $2^{l_{1} l_{2} \cdots l_{k}} \equiv 1(\bmod m)$ and the multiplicative 
order of $2 \bmod m$ would be odd. Hence we can choose $i$ so that the multiplicative order of $2 \bmod p_{i}^{\alpha_{i}}$ is even. Put $p=p_{i}$ and let $S_{p}$ be the Sylow $p$-subgroup of $T(G)$ (i.e., of $A$ ). Let $S_{2^{\prime}, p^{\prime}}$ be the product of all remaining Sylow subgroups of $A$. Theorem 2 ensures the existence of a nonzero nilpotent element $\eta$ in $\mathbb{Z}\left[S_{p} \times K_{8}\right]$.

Now we put: $H=G /\left(H_{2} \cdot S_{2^{\prime}, p^{\prime}}\right), \phi: G \rightarrow H$ is the natural homomorphism, $V=\phi\left(S_{p}\right) \simeq S_{p}, U=\phi(\mathcal{U} \mathbb{Z} G)$.

As in the previous case we prove that the assumptions of Proposition 9 are satisfied. This time we have the following diagram of natural homomorphisms:

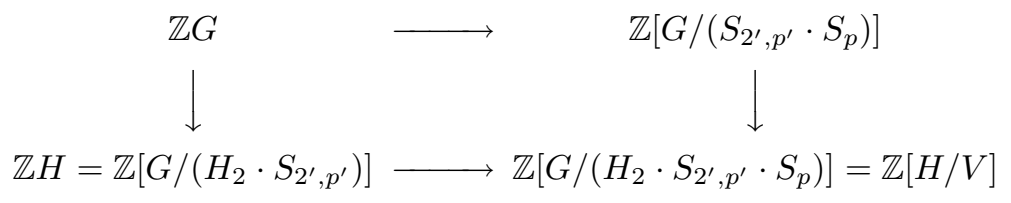

The torsion subgroup of $G /\left(S_{2^{\prime}, p^{\prime}} \cdot S_{p}\right)$ is $E \times K_{8}$. It is generally known that there are only trivial units in $\mathbb{Z}\left[E \times K_{8}\right]$. Because we assumed that there are no nontrivial, finite, not normal subgroups in $G$ we may apply Theorem 10 to conclude that there are only trivial units in $\mathbb{Z}\left[G /\left(S_{2^{\prime}, p^{\prime}} \cdot S_{p}\right)\right]$. Also $H<U$ and therefore $U$ is $p$-nice. Like before we can show that $\phi(\eta) \neq 0$ and $|\phi(C(\eta))|=\infty$. Applying Proposition 9 we obtain that $\mathcal{U} \mathbb{Z} G$ is not finitely generated.

The main result, Theorem 2, easily follows from the above theorem.

\section{ACKNOWLEDGEMENTS}

The author wishes to thank Dr. Zbigniew Marciniak for introducing him to the problematics considered here.

\section{REFERENCES}

1. M. Kargapolov and Yu. Merzljakov, Fundamentals of the theory of groups, Springer Verlag, 1979. MR 80k:20002

2. Z. Marciniak and S. K. Sehgal, Zassenhaus conjectures for infinite groups, Proceedings of the International Conference on Ring Theory, Miskolc '96, to appear.

3. M. Mirowicz, Units in group rings of the infinite dihedral group, Canad. Math. Bull. 34(1), (1991), pp. 83-89. MR 92g:16050

4. S. K. Sehgal, Topics in group rings, Marcel Dekker, 1978. MR 80j:16001

5. S. K. Sehgal, Units in integral group rings, Longman's, Essex, 1993. MR 94m:16039

Institute of Mathematics, Warsaw University, ul. Banacha 2, 02-097 Warszawa, Poland

E-mail address: lwiech@mimuw.edu.pl 